Bundesgesundheitsbl -

Gesundheitsforsch - Gesundheitsschutz

2000 • 43:432-437 @ Springer-Verlag 2000

\section{Zusammenfassung}

Gut zwei Drittel der Erwachsenen in Deutschland haben eine Sehhilfe. Durchschnittlich geht ein Brillenträger jedes dritte Jahr, ein Linsenträger jedes zweite Jahr zum Augenarzt. Kontaktlinsen kaufen fast nur Personen unter 40 Jahren. Der Markt für Brillen ist für die Käufer ab 45 Jahre besonders groß, denn die Weitsichtigkeit wird fast ausschließlich mit einer Brille korrigiert. Schwerhörigkeit ist bei 8\% der Erwachsenen verbreitet, aber nur 2,5\% der Bevölkerung im Alter von 18 bis 79 Jahren haben eine Hörhilfe. Die Schwerhörigen sind zu 59\% Männer und $41 \%$ Frauen. Die Schwerhörigkeit steigt steil mit dem Alter an. Eine Behinderung geben $12,4 \%$ der Befragten an. Es existieren sowohl regionale als auch geschlechtsspezifische Unterschiede. Mit zunehmendem Alter und der Anzahl der Krankheiten nimmt der Anteil der Behinderten deutlich zu.

\section{Schlüsselwörter}

Brille - Kontaktlinse - Sehfehler . Kurzsichtigkeit · Schwerhörigkeit · Hörhilfe · Behinderung

Leitthema: Bundes-Gesundheitssurvey

E. Bergmann · U. Ellert

Robert Koch-Institut, Berlin

\title{
Sehhilfen, Hörhilfen und Schwerbehinderung
}

er Begriff Behinderung ist sehr komplex und umfasst Einschränkungen in den körperlichen, seelischen und geistigen Funktionen, die nicht nur vorübergehend bestehen und dazu führen, dass den betroffenen Personen ihre unmittelbaren Lebensverrichtungen oder die Teilnahme am Leben in der Gesellschaft erschwert wird. Menschen mit Behinderungen bedürfen einer besonderen Förderung. Behinderungen können angeboren sein oder erst im Laufe des Lebens zum Beispiel durch Unfall oder Krankheiten entstanden sein. Sie können unterschiedlich schwer sein und unabhängig von ihrem Schweregrad subjektiv völligunterschiedlich bewertet und empfunden werden.

Zwei große Gruppen von überwiegend leichterer Behinderung sind die Seh- und Hörschäden, die mit Hilfsmitteln korrigierbar sind. Die Versorgung der Bevölkerung in Deutschland mit Hilfsmitteln ist jedoch nur teilweise bekannt. Nicht alle zum Kauf angebotenen Hilfsmittel werden von den verschiedenen Leistungsträgern bezahlt bzw. werden die Kosten nur anteilig von ihnen übernommen. Daher sind Statistiken der Leistungsträger auch nur von begrenzter Bedeutung für Fragestellungen zur Versorgung der Bevölkerung. Am Beispiel der Sehhilfen und Hörhilfen werden versorgungsepidemiologische Eckwerte genannt und epidemiologische Fragestellungen zu den Hilfsmitteln, den ihnen zugrunde liegenden gesundheitlichen Störungen und zur Behinderung allgemein erörtert, soweit sie auf der empirischen Basis des BundesGesundheitssurveys beruhen. Bei der
Interpretation der Ergebnisse ist zu beachten, dass am Survey nur die Personen teilgenommen haben, die dazu geistig und körperlich auch in der Lage waren. Schwerbehinderte Personen, die stationär oder in Heimen betreut werden müssen, wurden mit dieser Befragung nicht erreicht.

\section{Material und Methoden}

Die Auswertung beruht auf den Daten des Bundes-Gesundheitssurveys 1998, der aus der Befragung und Untersuchung von 7124 Personen einer mehrstufigen, geschichteten Bevölkerungsstichprobe der 18- bis 79-jährigen Einwohner Deutschlands bestand [1]. Sämtliche Berechnungen wurden so gewichtet, dass sie repräsentativ für die Bevölkerung sind. In Analogie zu den Faustregeln für Genauigkeitsangaben des Mikrozensus sollten hochgerechnete Differenzen bei Einwohnerzahlen von unter 500000 nicht interpretiert werden. Die Datenaufbereitung und Auswertung erfolgte in SPSS Version 9 [2]. Die generalisierten linearen Modelle, hier LogitAnalysen, wurden primär mit GLIM Version 4.09 [3] gerechnet. Die Irrtumswahrscheinlichkeit für die Parameterschätzungen der Modelle beträgt $5 \%$. Die Hypothesen konkretisierten sich erst im Verlauf der Modellierung, so dass - streng genommen - die LogitAnalysen im Sinne hypothesengenerierender Verfahren verwendet wurden.

Eckardt Bergmann

Robert Koch-Institut, Postfach 6502 80,

13302 Berlin 


\section{E. Bergmann · U. Ellert}

\section{Seeing Aids, Hearing Aids, and Disabilities}

\section{Summary}

More than $2 / 3$ of German adults are using seeing aids. Individuals who are wearing eyeglasses are consulting their opthalmic practitioner about every 3rd year while persons with contact lenses see their practitioner every 2 nd year. Almost only people under age 40 are buying contact lenses. The market for spectacles is very large for consumers older than 45 years since hypermetropia is mainly corrected with eyeglasses in this age group. The prevalence of hard of hearing is $8 \%$ for adults and is increasing with age, but only $2,5 \%$ of the study population are equipped with hearing aids. The distribution between males and females is $59 \%$ vs. $41 \%$ for hard of hearing. $12,4 \%$ of the interviewed persons report a handicap. There are regional and gender-specific differences. The proportion of handicapped person raises with increasing age and counts of diseases.

\section{Keywords}

Seeing aids - ametropia · myopia - Officulties in hearing aids $\cdot$ disability

\section{Sehfehler und Versorgung mit Sehhilfen}

Die Versorgung der Bevölkerung mit Sehhilfen wird zu einem Teil von den Krankenkassengetragen. Aus der Jahresstatistik 1997 der GKV [4] sind die Ausgaben für Sehhilfen von insgesamt 1,452 Milliarden DM für 71,5 Millionen Versicherten zu entnehmen. Somit entfallen jährlich pro Versichertem 20,31 DM für Sehhilfen. Den größten Anteil der Kosten für eine Sehhilfe dürfte der Versicherte bei der Zuzahlung jedoch selbst tragen. Unbekannt ist der Anteil der Versicherten bzw. in der Gesamtbevölkerung, die eine Sehhilfe haben. Erst recht sind die Informationen über Altersverteilungund Art des Sehfehlers sowie weitere bedeutende Einflussfaktoren auf die wichtigsten Maßnahmen zur Korrektur der Sehfehler nicht bekannt. Aus den Daten des Bundes-Gesundheitssurveys 1998 sollen daher Eckdaten zur Versorgungsepidemiologie mit Sehhilfen genannt werden.

\section{„Etwa zwei Drittel der Erwachsenen in Deutschland benötigen eine Sehhilfe!"}

Hochgerechnet auf die Bevölkerung im Alter zwischen 18 und 79 Jahren gibt es 40 Millionen Personen (64,4\% der Bevölkerung), die eine Brille tragen, und 2,2 Millionen Personen (3,5\%) mit Kontaktlinsen, die zum Teil zusätzlich eine
Brille haben. Somit tragen zwei Drittel der Erwachsenen eine Sehhilfe. Gut die Hälfte der Kontaktlinsenträger war im letzten Jahr beim Augenarzt. Die Brillenträger gingen nur zu einem Drittel zum Augenarzt. Mit anderen Worten, ein Brillenträger geht durchschnittlich alle drei Jahre zum Augenarzt, eine Person mit Kontaktlinsen jedes zweite Jahr [5]. Die Inanspruchnahme eines Optikers, der ggf. ohne ärztliche Verordnung Sehhilfen verkaufen kann, wurde im Bundes-Gesundheitssurvey nicht erfragt.

Die Verteilung der Sehhilfen nach dem Alter in der Bevölkerung zeigt Abb. 1. Personen im Alter von unter 45 Jahren benötigen seltener eine Sehhilfe. Ab 50 Jahren haben fast alle Einwohner eine Sehhilfe. Für Brillen besteht der größte Markt bei Personen ab 45 Jahren. Kontaktlinsen können fast nur im Altersbereich von 20 bis unter 40 Jahren verkauft werden.

\section{Epidemiologie der Sehfehler}

Der Versorgungsgrad mit Sehhilfen hängt stark von der Art der Sehfehler ab. Unterscheidet man nur die drei Sehfehler "Kurzsichtigkeit", "Weitsichtigkeit" und "Sonstiges", die auf den Selbstangaben des Probanden beruhen, so kann man davon ausgehen, dass in der Regel die Kurzsichtigkeit mit Brille oder Kontaktlinse ausreichend korrigiert wird. Bei Weitsichtigkeit wird fast nur mit einer Brille (Lesebrille) korrigiert (vgl. Tabelle 1). Weitsichtigkeit (Presbyopie) ist der altersbedingte Elastizitätsverlust der Linse. Umgangssprachlich werden alle

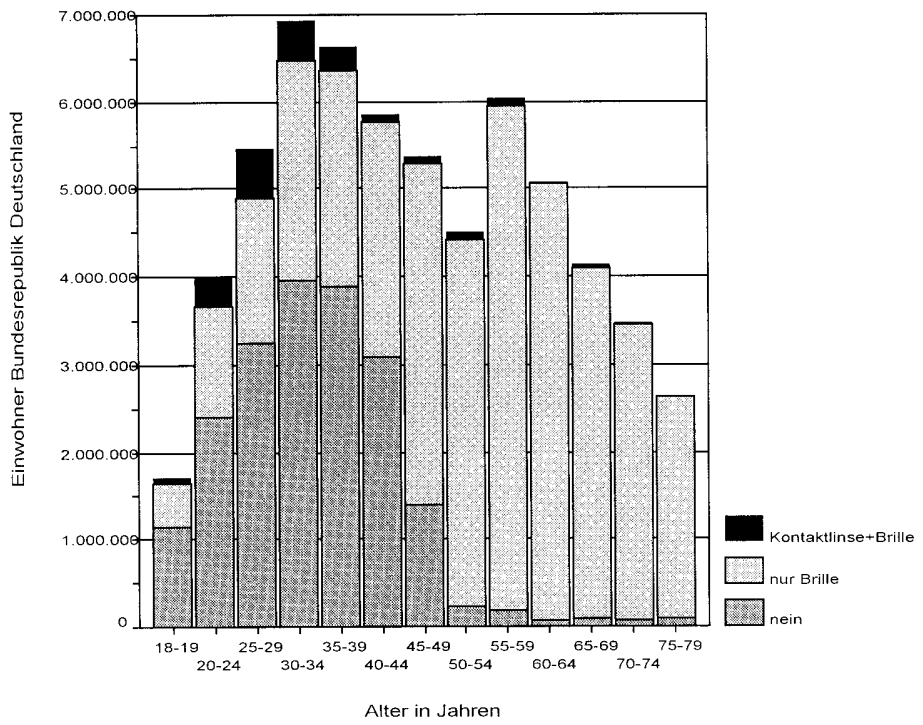


Tabelle 1

Sehhilfen nach Art der Sehfehler

nur Brille Kontaktlinse etc. keine Sehhilfe

\begin{tabular}{llrr}
\hline kurzsichtig & $89,9 \%$ & $6,8 \%$ & $3,3 \%$ \\
weitsichtig & $96,0 \%$ & $1,7 \%$ & $2,3 \%$ \\
anderer Sehfehler & $73,6 \%$ & $4,9 \%$ & $21,5 \%$
\end{tabular}

Tabelle 2

Berufliche Belastung und Kurzsichtigkeit

\begin{tabular}{lccc} 
& kurzsichtig & nicht kurzsichtig & gesamt \\
\hline weniger belastet & $41,2 \%$ & $58,8 \%$ & $100 \%$ \\
sonst belastet & $46,0 \%$ & $54,0 \%$ & $100 \%$ \\
höher belastet & $48,5 \%$ & $51,5 \%$ & $100 \%$
\end{tabular}

Formen der Übersichtigkeit (Hypermetropie) ebenfalls als Weitsichtigkeit verstanden.

Kurzsichtigkeit (Myopie) ist die Fehlsichtigkeit (Ametropie) weiter entfernt liegende Objekte nicht scharf genug sehen zu können, da der Brennpunkt ins Auge fallender parallelerStrahlen vor der Netzhaut liegt. Die Ursachen für diesen Refraktionsfehler des Auges können vor allem in der Brechung der Hornhaut, in der Brechung der Linse, in der Achsenlänge des Auges, in Formanomalien oder auch an den verschiedenen Mediendichten liegen [6]. Die Ätiologie der Myopie ist vermutlich multifaktoriell. Genetische Faktoren scheinen bedeutender zu sein als Einflüsse durch Sehgewohnheiten [7, 8]. Die Auffassungen, inwieweit Kurzsichtigkeit vererbt oder erworben ist, gehen weit auseinander. Für die Vererbungstheorie stehen beispielhaft Jaeger und Hammerstein [9], die feststellen, dass “... bei eineiigen Zwillingen trotz verschiedener Beanspruchung der Augen gleiche Refraktion entsteht, während sich bei zweieiigen Zwillingen trotz gleicher Tätigkeit die Refraktion verschieden entwickelt" (S. 629). Dass Einflüsse der Umwelt, insbesondere längere Schulbildung,
Lesen, Bildschirmarbeit neben biologischen Determinanten bei der Entwicklung von Myopie eine Rolle spielen, behaupten die meisten Autoren [10, 11, 12, 13]. Aber auch, dass die Entwicklung der Kurzsichtigkeit durch Bildschirmarbeit im Vergleich zu normaler Bürotätigkeit nicht stärker ist, wird belegt [14]. Über Kausalitäten oder inwieweit Kurzsichtigkeit erblich bzw. erworben ist, kann mit den statistischen Analysen anhand der Daten des Bundes-Gesundheitssurveys kaum eine Aussage gemacht werden.

Bildet man Gruppen mit unterschiedlicher Belastung beim Sehen im Nahbereich aufgrund der Angaben über die Ausübung der jetzigen bzw. letzten beruflichen Tätigkeit und über die Bildung, so ergeben sich mit der Steigerung der Belastung höhere Prävalenzen für Kurzsichtigkeit (s. Tabelle 2). Zur Gruppe der geringer Belasteten gehören Personen mit den Berufsgruppen Landwirte, Tierzüchter, Fischereiberufe, landwirtschaftliche Arbeitskräfte, Tierpfleger, Forst- und Jagdberufe, Bergleute, Mineral-, Erdöl-, Erdgasgewinner und die Bauberufe. Die Gruppe der höher Belasteten umfasst Glasbläser (vor der Lampe), Feinmechaniker, Uhrmacher,

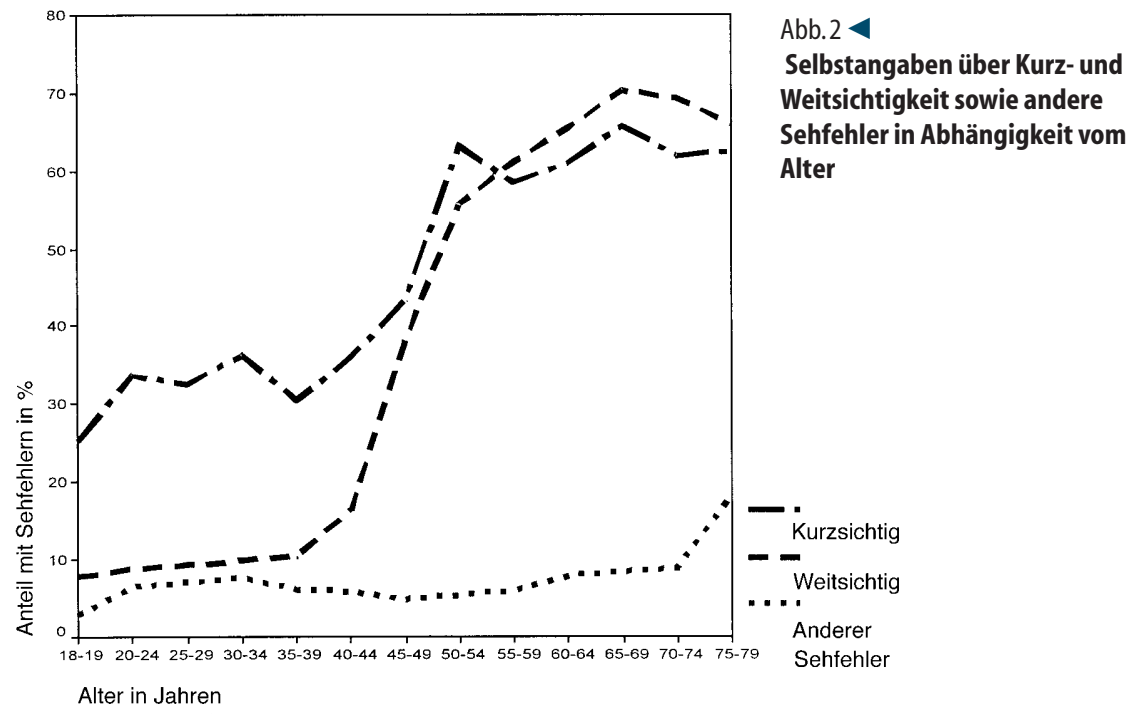

Werkzeugmacher, Metallfeinbauer (mit Edelmetallschmieden, Zahntechnikern, Augenoptikern, Musikinstrumentenbauern, Puppenmachern, Präparatore etc.).Zusätzlich wurden die Schüler zwischen 18 und unter 20 Jahren, die noch keinen Schulabschluss hatten, die Studenten und die Akademiker (mit Abitur als allgemeinem Schulabschluss und dem Hochschulabschluss) zur Gruppe der höher Belasteten gezählt. Der Rest der Befragten bildet die Gruppe der sonstig Belasteten.

Die Kurzsichtigkeit ist bei Beamten, die Beihilfe erhalten und privat Versicherten weiter verbreitet als bei Versicherten der Innungs- und Ortskrankenkassen. Dies könnte eine Folge der unterschiedlichen beruflichen Belastung beim Sehen im Nahbereich sein. Insgesamt unterscheiden sich die Prävalenzen der Kurzsichtigkeit nach der beruflichen Belastung jedoch nur wenig.

Frauen sind mit gut 50\% Prozent häufiger Kurzsichtig als Männer mit gut 40\%. Ebenso steigt mit der Anzahl der genannten Krankheiten im Leben auch die Kurzsichtigkeit; dabei wurden Sehfehler nicht als Krankheit eingerechnet. Bei Kurzsichtigen kann auf allen Skalen des SF- $36^{1}$ zur subjektiven gesundheitsbezogenen Lebensqualität ein etwas niedriger Wert festgestellt werden, der bei körperlicher Funktionsfähigkeit und körperlicher Rollenfunktion sogar um $5 \%$ niedriger liegt als bei Personen, die keine Sehhilfen benötigen. Der Zerssenwert $^{2}$ aus der Beschwerdenliste liegt bei Kurzsichtigen mit 17,6 weit höher als bei Personen, deren Wert nur 14,3 beträgt ein Wert nahe an der Testnorm der Beschwerdenliste.

Die Abhängigkeit des Sehfehlers und damit auch der Korrekturmaßnahme vom Alter ist besonders stark (vgl.

\footnotetext{
${ }^{1}$ Zum SF-36 siehe auch [18]

${ }^{2}$ Zur Verwendung der Beschwerdenliste nach v.Zerssen im Bundes-Gesundheitssurvey siehe [19]
} 


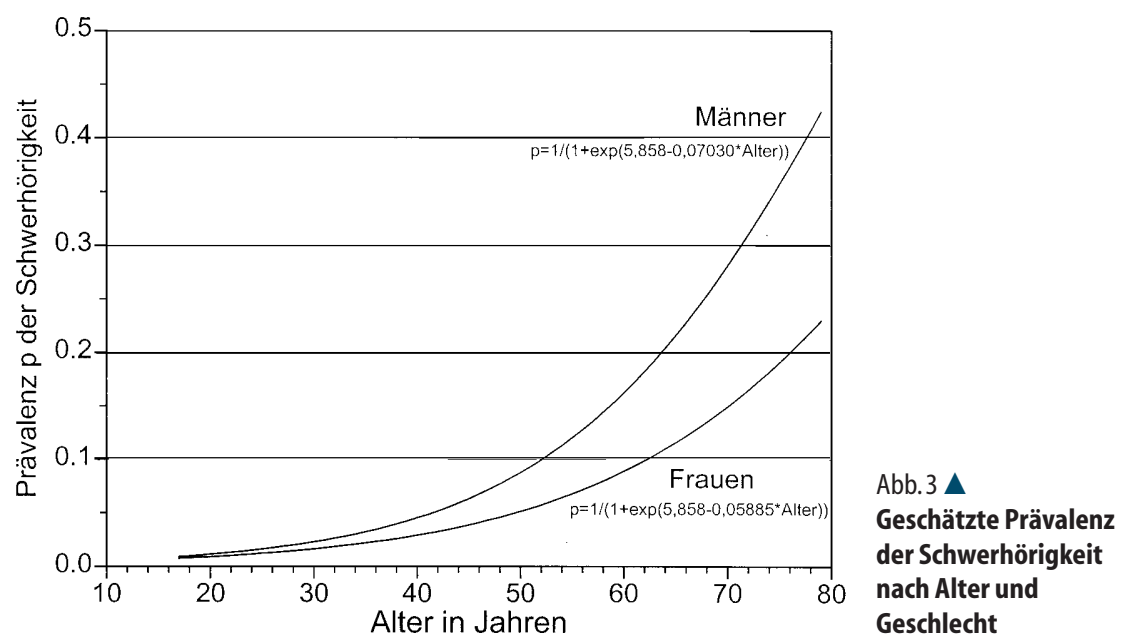

Abb. 2). Unter Weitsichtigkeit leiden bis 40 Jahre weniger als 10\%. Die Weitsichtigkeit steigt mit dem Alter zunächst sehr schnell und dann ab ca. 50 Jahre langsamer bis auf über $60 \%$ in der Bevölkerung. Andere Sehfehler verbleiben mit gut 5\% im Altersbereich bis unter 60 Jahre relativ selten und steigen erst im hohem Alter steil an. Nach den durch Selbstangabe der Probanden erhobenen Ergebnissen des Bundes-Gesundheitssurveys verdoppelt sich auch die Kurzsichtigkeit mit zunehmendem Alter ab ca. 45 Jahre von vorher 30\% auf 60\% der Bevölkerung Deutschlands. Diese Zunahme der Kurzsichtigkeit mit dem Alter in der Erwachsenenpopulation steht jedoch im Gegensatz zu bisherigen epidemiologischen Untersuchungen zur Myopie [15, 16, 17].

Die wahrscheinlichste Erklärung für den unerwarteten Befund einer deutlichen altersabhängigen Zunahme der Kurzsichtigkeit dürfte darin liegen, dass die Begriffe Kurzsichtigkeit und Weitsichtigkeit von einem erheblichen Teil der Probanden - besonders aus der Alterskohorte - falsch interpretiert wurde, d.h. dass Kurzsichtigkeit im Sinne einer Krankheit/Behinderung als schlechtes Sehen im Nahbereich verstanden worden sein könnte. Andere, aber weniger plausible Erklärungen für den starken Anstieg der Kurzsichtigkeit mit dem Alter könnten sein:

D Bei einem Teil der Bevölkerung werden erst nach dem Eintritt der Weitsichtigkeit im höherem Alter Beeinträchtigungen in der Sehleistung wahrgenommen, die zur Inan- spruchnahme eines Augenarztes oder Optikers führen. Im Rahmen des Sehfähigkeitstests wird dann eine eventuell schon länger vorhandene, vermutlich geringe, Kurzsichtigkeit diagnostiziert.

D Die bisherigen epidemiologischen Untersuchungen zur Myopie erwachsener Personen orientieren sich an den Untersuchungen von Kindern und Jugendlichen, die den größten Anteil an Studien ausmachen. Daher könnte ein solcher Effekt im späteren Alter übersehen worden sein.

Multiple Logit-Analysen, die hier nicht weiter ausgeführt werden sollen, bestätigen das vermutete, vom Alter abhängige unterschiedliche Verständnis des Begriffs Kurzsichtigkeit.

Um die Unsicherheit zu beseitigen, ob und inwieweit die Probanden die Begriffe Kurzsichtigkeit und Weitsichtigkeit richtig verstehen, wäre es sinnvoll, in einem neuen Gesundheitssurvey eine Frageformulierung zu wählen, die weniger Anlass zu Missverständnissen bietet. Zu empfehlen wäre außerdem, zumindest probeweise ein Refraktometer einzusetzen, mit dem die Stärke einer vorhandenen Brille gemessen werden kann. Eine erwünschte Bestimmung der Sehschärfe dürfte hingegen für einen Survey $z u$ aufwendig sein.

\section{Versorgung mit Hörhilfen und Schwerhörigkeit}

Für Hörgeräte wurden 1997 insgesamt 722 Millionen DM von der GKV ausge- geben bzw. gut 10 DM pro Versichertem. Auch hier muss mit einer erheblichen Zuzahlung gerechnet werden. Hörhilfen benötigt ein weit geringerer Anteil in der Bevölkerung als Sehhilfen. Nach Selbstangaben im Bundes-Gesundheitssurvey 1998 bezeichnen sich 8\% der erwachsenen Bevölkerung bis unter 80 Jahren als schwerhörig, von denen wiederum gut $30 \%$ ein Hörgerät besitzen. Insgesamt nutzen 2,5\% eine Hörhilfe. In ärztlicher Behandlung bei einem Facharzt für Hals/Nasen/Ohren waren im letzten Jahr 31\% der Schwerhörigen, während von den Befragten mit normalem Hörvermögen nur $12 \%$ beim HNOArzt waren.

\section{„Etwa 8\% der erwachsenen Bevölkerung bis unter 80 Jahren bezeichnen sich als schwerhörig, von diesen wiederum besitzen gut 30\% ein Hörgerät."}

Von den Schwerhörigen sind 59\% Männer und 41\% Frauen. Die Schwerhörigkeit steigt steil mit dem Alter von knapp $2 \%$ für die unter 30 -Jährigen bis über $30 \%$ für die ab 60-Jährigen. Der Altersanstieg ist für die Männer steiler als für die Frauen (vgl. Abb.3).

Zusätzliche starke Einflussgrößen auf die Prävalenz $p$ der Schwerhörigkeit ${ }^{1}$ sind die Anzahl der Krankheiten im Leben, der Schichtindex und die ZerssenBeschwerdenliste

Schwerhörigkeit ist eher ein Problem der Unterschicht und wächst mit der Anzahl der sonstigen Krankheiten im Leben. Die Zunahme der Beschwerden mit der Prävalenz der Schwerhörigkeit könnte als Hinweis auf mögliche Persönlichkeitsveränderungen, die durch Schwerhörigkeit entstehen können, verstanden werden. Andere Einflussgrößen auf die Schwerhörigkeit verlieren ihre Bedeutung, wenn Alter, Geschlecht, Anzahl Krankheiten im Leben, soziale Schicht und Beschwerdenliste gleichzeitig berücksichtigt werden. Regionale Unterschiede zwischen Ost und West, Bundesländern und Siedlungsdichte sind bei zweiseitiger Betrachtung erkennbar - eine höhere

\footnotetext{
${ }^{1} \mathrm{p}=\frac{1}{1+e^{\left.\left.-\left(-6,042+0,07106^{*} \text { Anzahl/Krankheiten }+0,03857^{*} \text { Zerssenwert-0,04529*SChichtindex }+0,06671^{*} \text { Alter(Männer }\right)+0,05019^{*} \text { Alter(Frauen }\right)\right)}}$
} 


\section{Leitthema: Bundes-Gesundheitssurvey}

Prävalenz liegt tendenziell im Westen, nördlichen und westlichen Bundesländern und bei höherer Bevölkerungsdichte vor - sind aber im Simultanmodell nicht relevant. Der SF-36 hat zunächst eine starke Beziehung zur Schwerhörigkeit, hier besonders bei körperlicher Funktionsfähigkeit und Rollenfunktion, der jedoch im Simultanmodell nicht mehr signifikant ist. Vermutlich ist der SF-36 nur solange von statistischer Bedeutung für körperliche $\mathrm{Be}$ einträchtigungen, die nicht direkt im SF36 gemessen werden, bis Alter und Geschlecht ausreichend in Modellen berücksichtigt worden sind. Dieses Ergebnis wird bekräftigt in der o.g. Modellierung der Kurzsichtigkeit. Eine gesunde Lebensweise, gemessen durch (überwiegend) vegetarische Lebensweise, Nichtrauchen und viel körperliche Aktivität zeigt zunächst einen positiven Effekt auf die Hörfähigkeit, jedoch bei der Berücksichtigung der intervenierenden Variablen Alter und Geschlecht sind diese Effekte ebenfalls nicht mehr signifikant. Keine Rolle spielen Lärm in der Wohnumgebung und Lärm, Staub, Gase etc. bei der Arbeit.

\section{Behinderung}

Als Behinderung wird die Auswirkung einer nicht nur vorübergehenden Funktionsbeeinträchigung, die auf einem regelwidrigen körperlichen, geistigen oder seelischen Zustand beruht, bezeichnet [20]. Im Bundes-Gesundheitssurvey gaben $12,4 \%$ der Probanden an, dass bei ihnen eine vom Versorgungsamt amtlich anerkannte Behinderung besteht. Unterschiede ergeben sich sowohl im Altersverlauf als auch zwischen den Geschlechtern. Mit zunehmendem Alter nimmt der Anteil der Behinderten unter den Probanden zu. Männer sind mit $13,9 \%$ häufiger behindert als Frauen $(10,3 \%)$.

\section{"12,4\% der Probanden geben an, dass bei ihnen eine vom Versorgungsamt amtlich anerkannte Behinderung besteht, von diesen sind mehr als 60\% schwerbehindert."}

Eine mehr als 50\%ige Behinderung gilt als Schwerbehinderung im Sinne des

Tabelle 3

Logistische Regression - Einflussfaktoren für Schwerbehinderung

\begin{tabular}{|c|c|c|c|}
\hline \multicolumn{4}{|c|}{ Männer } \\
\hline Einflussfaktoren & Odds-Ratio (95\% Cl) & Konfidenzintervall 95\% & Signifikanz \\
\hline Alter in Jahren & 1,06 & $1,05-1,07$ & $* * *$ \\
\hline West & 1,64 & $1,12-2,40$ & $*$ \\
\hline Ost & 1 & Referenz & \\
\hline Unterschicht & 1 & Referenz & \\
\hline Mittelschicht & 1,04 & $0,73-1,48$ & n.s. \\
\hline Oberschicht & 0,62 & $0,40-0,94$ & * \\
\hline Anzahl von Krankheiten & 1,31 & $1,24-1,37$ & $* * *$ \\
\hline \multicolumn{4}{|c|}{ Frauen } \\
\hline Einflussfaktoren & Odds-Ratio (95\% Cl) & Konfidenzintervall 95\% & Signifikanz \\
\hline Alter in Jahren & 1,04 & $1,02-1,05$ & $* * *$ \\
\hline Anzahl von Krankheiten & 1,3 & $1,25-1,34$ & \\
\hline $\begin{array}{rl}* * * * * & p<0,001 \\
* * & p<0,005 \\
* & p<0,05 \\
& p>=0,05\end{array}$ & & & \\
\hline
\end{tabular}

Schwerbehindertengesetztes. Danach können mehr als $60 \%$ der untersuchten behinderten Personen als schwerbehindert bezeichnet werden. Das Vorliegen einer Schwerbehinderung ist offensichtlich stark altersabhängig. Bei den Männern ist der Zuwachs mit dem Alter noch deutlich höher als bei den Frauen (Abb. 4).

Weitere Zusammenhänge ergeben sich zwischen dem Vorliegen einer Behinderung oder Schwerbehinderung und der sozialen Schicht sowie dem Leben in Ost- oder Westdeutschland. Männer der Unter- und Mittelschicht sind zu knapp 10\% schwerbehindert, in der Oberschicht sind es 7,2\%. Bei den Frauen sind es $8,6 \%$ in der Unter- und $5,2 \%$ in der Oberschicht. Männer im Westen haben mit 9,9\% einen höheren Schwerbehindertenanteil als diejenigen im Osten mit 6,2\%. Je mehr Krankheiten im Laufe des Lebens durchgemacht wurden, desto höher ist auch die Wahrscheinlichkeit behindert zu werden.

Tabelle 3 zeigt die gegenseitige Wechselwirkung verschiedener Risikofaktoren auf das Auftreten von Schwerbehinderung. In das Modell gingen Alter, soziale Schicht, Region und die Krankheitsanzahl unabhängig von ihrem Schweregrad ein. Da sich in den bivariaten Analysen große Geschlechtsunterschiede ergaben, wurden die Auswertungen für Männer und Frauen getrennt vorgenommen. Als stabile Einflussgrö-
Prävalenz von Schwerbehinderung nach Alter und Geschlecht

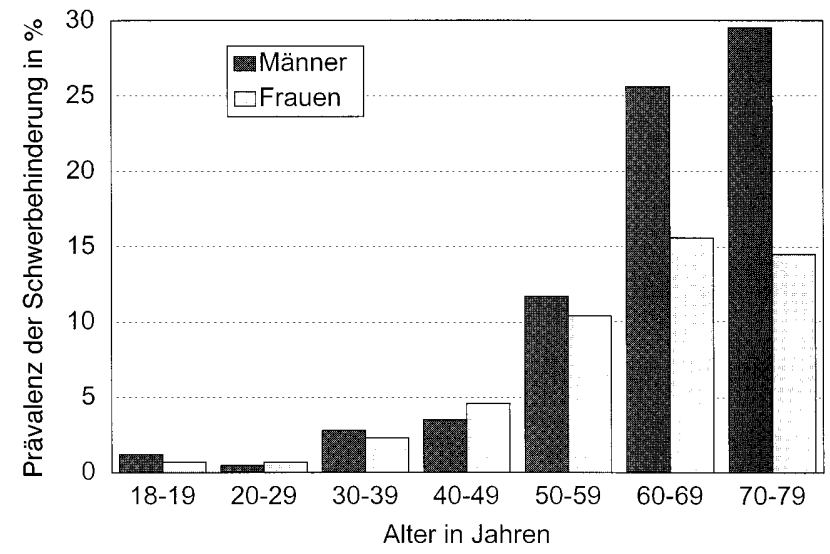




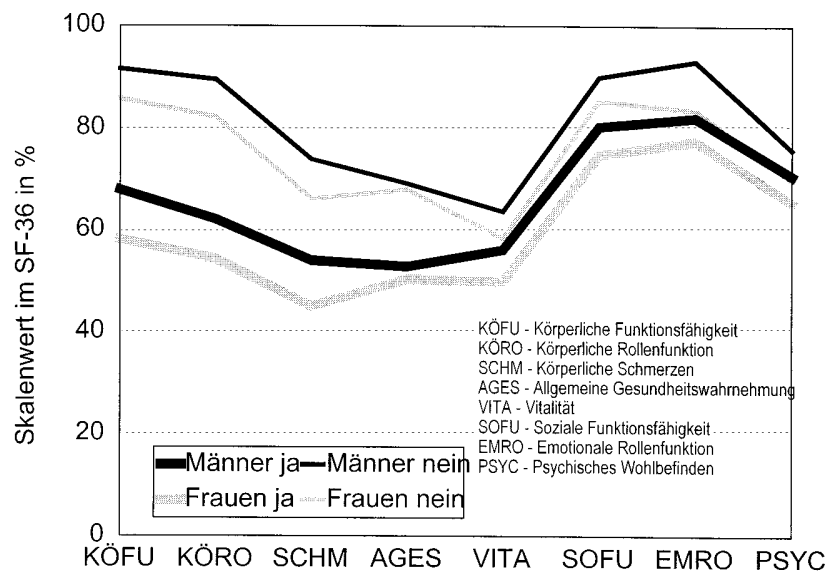

Abb. $5<$

SF-36 und Behinderung

ßen erweisen sich bei den Männern Alter, soziale Schicht, das Leben in Ostoder Westdeutschland und die Anzahl der Krankheiten. Die Wahrscheinlichkeit, schwerbehindert zu sein erhöht sich mit zunehmendem Alter und steigender Krankheitsanzahl. Der Einfluss der territorialen Zugehörigkeit und der Zugehörigkeit zu einer sozialen Schicht ist relativ groß. Diese beiden Größen lieferten für die Frauen keinen Beitrag zur Erklärung des Modells, hier blieben der Einfluss des Alters und der Anzahl der Krankheiten erhalten.

Als Maß für die gesundheitsbezogene Lebensqualität wurde im Survey der SF-36 Fragebogen eingesetzt. Die Abbildung 5 verdeutlicht, dass sich Menschen mit Behinderungen in ihrer Lebensqualität gegenüber Nichtbehinderten beeinträchtigt fühlen. Kausale Zusammenhänge können mit unseren Daten aber nicht geklärt werden. Männer und Frauen ohne Behinderungen erreichen in allen Skalen des SF-36 höhere Werte als diejenigen mit Behinderungen.

\section{Literatur}

1. Thefeld W, Stolzenberg H, Bellach BM (1999) Bundes-Gesundheitssurvey: Response, Zusammensetzung der Teilnehmer und Non-Responder-Analyse. Gesundheitswesen Sonderheft 2,61:603-608

2. http://www.spss.com

3. http://www.nag.co.uk

4. Bundesministerium für Gesundheit (1999)

Die gesetzliche Krankenversicherung in der Bundesrepublik Deutschland im Jahre 1997 - Statistischer und finanzieller Bericht. Bonn, Eigenverlag

5. Bergmann E, Kamtsiuris P (1999) Inanspruchnahme medizinischer Leistungen. Gesundheitswesen Sonderheft 2,61:138-144

6. Whitmore WG (1992) Congenital and developmental myopia. Eye 6:361-365

7. Pacella R,McLellan J, Grice K, del Bono EA, Wiggs JL, Gwiazda JE (1999) Role of genetic factors in the etiology of juvenile-onset myopia based on a logitudinal study of refractive error. Optometry And Vision Science 76:381-386

8. Wu MM, Edwards MH (1999) The effect of having myopic parents: an analysis of myopia in three generations (see comments). Optometry And Vision Science 76: 387-392

9. Jaeger W, Hammerstein W (1992) Vererbung von Augenleiden und Anomalien des Auges. In: Pau H (Hrsg) Lehrbuch der Augenheilkunde. Fischer, Stuttgart, Jena, New York, S 617-643

10. Saw SM, Chia SE, Chew SJ (1999) Relation between work and myopia in Singapore women. Optometry And Vision Science 76: 393-396

11. Saw SM, Katz J, Schein OD, Chew SJ, Chan TK (1996) Epidemiology of myopia. Epidemiologic Reviews 18: 175-187

12. Angle J, Wissmann DA (1980) The epidemiology of myopia. American Journal of Epidemiology 111:220-228
13. Wilson A, Woo G (1989) A review of the prevalence and causes of myopia. Singapore Medical Journal 30:479-484

14. Rechichi C, Scullica L (1996) Trends regarding myopia in video terminal operators. Acta Ophthalmologica Scandinavica 74:493-496

15. Sperduto RD, Hiller R, Podgor MJ, Freidlin V, Milton RC, Wolf PA, Myers RH, D'Agostino RB, Roseman MJ, Stockman ME, Wilson PWF (1996) Familial aggregation and prevalence of myopia in the Framingham Offspring Eye Study. Archives of Opthalmology 114 : 326-332

16. Mutti DO, Bullimore MA (1999) Myopia: an epidemic of possibilities? (editorial). Optometry And Vision Science 76:257-258

17. Richler A, Bear JC (1980) The distribution of refraction in three isolated communities in Western Newfoundland. American Journal Of Optometry And Physiological Optics 57: 861-871

18. Ellert U, Bellach BM (1999) Der SF-36 im Bundes-Gesundheitssurvey - Beschreibung einer aktuellen Normstichprobe. Gesundheitswesen Sonderheft 2, 61:184-190

19. Bellach BM, Knopf H, Thefeld W (1998) Der Bundes-Gesundheitssurvey 1997/1998. Gesundheitswesen Sonderheft 2,60:59-68

20. Hauptfürsorgestelle Berlin (Hrsg) (1993) Behinderung und Ausweis. Stand September 1993. Eigenverlag, Berlin 